\title{
Juvenile idiopathic arthritis: An observational study
}

\author{
K A Nihal Gunatillaka ${ }^{1}$, M K Kelum de Silva ${ }^{2}$ \\ Sri Lanka Journal of Child Health, 2007; 36: 96-101
}

(Key words: Juvenile idiopathic arthritis, observational study)

\begin{abstract}
Objectives To assess demography, types of arthritis, treatment modalities and their side effects in patients with arthritis.
\end{abstract}

Method An interviewer administered questionnaire was used obtaining information from both the patient and carer.

Results Four main types of arthritis were identified viz. polyarticular onset JIA (20 patients), systemic onset JIA (18 patients), enthesitis related arthritis (13 patients) and pauciarticular onset JIA (10 patients).

\section{Introduction}

Juvenile idiopathic arthritis (JIA) is an uncommon disease entity. Its prevalence in United Kingdom is estimated to be 50 cases per 100,000. Unfortunately, we have no epidemiological data for Sri Lanka. The term JIA encompasses several types of arthritis, viz. systemic onset, pauciarticular, polyarticular, enthesitis-related, psoriatic and other. The rheumatology clinic at Lady Ridgeway Children's Hospital has one of the largest collections of patients with JIA in Sri Lanka. We decided to carry out a study on patients with JIA attending this clinic from December 2004 to February 2005.

\section{Objectives}

- $\quad$ To assess demography of patients with arthritis.

- To assess types of arthritis, their characteristics and complications that patients have developed.

- To assess treatment modalities and their side effects.

\section{Method}

An interviewer administered questionnaire was used. Information was obtained from both the patient and the carer. Information was also obtained from clinic notes. Contractures and deformities were assessed by the consultant rheumatologist.

\footnotetext{
${ }^{1}$ Consultant, ${ }^{2}$ Senior Registrar in Rheumatology \& Rehabilitation, Lady Ridgeway Hospital for Children, Colombo.
}

(Received on 29 August 2006)

\section{Results}

Four main types of arthritis were identified viz. polyarticular onset JIA (20 patients), systemic onset JIA (18 patients), enthesitis-related arthritis (13 patients) and pauciarticular onset JIA (10 patients). There was also one patient with psoriatic arthritis.

\section{Polyarticular onset JIA}

One third of all patients in our study group (20 patients) belonged to this category. The female to male ratio was $3: 2$. There were 16 Sinhalese, 3 Muslims \& 1 Tamil patient. Ten patients were from the western province. Others were scattered throughout the island. The age at onset peaked between 4 and 8 years. Four patients gave a family history of rheumatoid arthritis. All 4 patients were female and three of them had their onset between 7 and 8 years of age.

Clinical features - In our series, 15 patients had fever at onset. Ten patients had low grade fever $\left(<39^{\circ} \mathrm{C}\right)$, in 9 lasting less than a week and in one patient for 1 month. Five patients had high grade fever $\left(>39^{\circ} \mathrm{C}\right), 2$ of them for more than a month. Three patients had a rash at the onset. Two patients had hepatomegaly, one had splenomegaly and two had lymphadenopathy. Most commonly involved joints initially were knees, wrists, ankles and small joints of the hands as seen in table 1.

Table 1 Initial joint involvement

\begin{tabular}{|l|c|}
\hline Joint/s & No. of patients \\
\hline Knee & 14 \\
\hline Wrist & 12 \\
\hline Ankle & 11 \\
\hline Proximal interphalangeal & 09 \\
\hline Metacarpophalangeal & 08 \\
\hline Cervical spine & 02 \\
\hline Hip & 01 \\
\hline
\end{tabular}

Investigations - All 20 patients had a raised erythrocyte sedimentation rate (ESR), more than half having an ESR of more than $100 \mathrm{~mm} / 1^{\text {st }}$ hour. Leucocytosis was found in 11 patients while 6 had normal white blood cell (WBC) counts. Anaemia was found in 7 patients while 8 had normal haemoglobin levels. Thrombocytosis was found in 8 patients while 7 had normal platelet counts. Rheumatoid factor test had been done in 18 patients and was positive in 2. One of them had the disease onset at 8 years and the 
other at 11 years. One patient is now in remission without joint deformities while the other continues to have active disease with joint deformities. Both patients have had the disease for 2 years. Antinuclear antibody (ANA) test was done in 16 patients and was negative in all of them.

Complications - One patient, who had had active disease for 7 years, developed iridocyclitis. Another, who had had active disease for 4 years, developed bilateral keratitis. Twelve patients had developed joint deformities over the course of time. All these patients have had active disease for at least 2 years before developing deformities. Eleven patients had developed joint ankylosis. Apophyseal joint fusion of the cervical spine was seen in the radiographs of 2 patients who have had active disease for more than 3 years. Atlanto-axial subluxation was seen in three patients who have had the disease lasting for more than 6 years. Avascular necrosis of hips was detected in 1 patient. Heights \& weights of the patients were compared with standard growth charts of Sri Lankan children and those who were below the $3^{\text {rd }}$ centile for both height and weight were considered as having growth retardation. Fourteen patients had growth retardation.

Medications - Eighteen patients have had prednisolone during the course of their illness. Five patients had been on steroids for 1 year or less whilst 13 were given steroids for more than 2 years, six of them for more than 5 years. All 20 patients were on methotrexate (MTX). In all but one patient MTX was started at this clinic. Only 5 patients had been on MTX for 3 or more years. Six patients were on sulphasalazine. Three patients who had refractory disease were given levamisole in addition to steroids, MTX and sulphasalazine. Three patients were on chloroquine. Ibuprofen and diclofenac sodium were the most popular non steroidal anti inflammatory drugs (NSAIDS) used. The adverse effects encountered by this subset of patients are shown in table 2.

Table 2

Adverse effects of drugs

\begin{tabular}{|l|c|}
\hline \multicolumn{1}{|c|}{ Adverse Effect } & No. of children \\
\hline Gastric irritation & 04 \\
\hline Haematemesis \& melaena & 01 \\
\hline Liver enzyme abnormalities & 01 \\
\hline Bone marrow suppression & 02 \\
\hline Steroid induced hypertension & 01 \\
\hline Osteoporosis (x-ray changes) & 02 \\
\hline
\end{tabular}

Outcome - Nine of the 20 patients are in remission although 7 of them are still on drugs. 6 patients have active disease 5 years after the onset. The 2 with complete remission had remitted within 2 years of onset of the disease.

\section{Systemic onset JIA}

There were 18 patients with systemic onset disease, accounting for $30 \%$ of the study population'. Eleven were males and 7 were females. Ethnic distribution was roughly similar to that of general population with 12 Sinhalese, 3
Tamils, 2 Muslims and 1 Burgher patient. In 17 patients the onset was under 6 years and peaked at 3 to 4 years.

Clinical Features - All patients had high grade fever $\left(>39^{\circ} \mathrm{C}\right)$ at the onset which is the hallmark of systemic onset JIA. The duration of fever varied but 8 patients had high fever for at least 2 months. Rash occurred at onset in 10 patients. Hepatomegaly was found in 11 patients, splenomegaly in 9 and lymphadenopathy in 11. Five patients reported having early morning joint stiffness. The pattern of initial joint involvement in this group of patients is shown in table 3 .

Table 3

Pattern of initial joint involvement

\begin{tabular}{|l|c|}
\hline Joint/s & No. of patients \\
\hline Knee & 08 \\
\hline Wrist & 08 \\
\hline Cervical spine & 06 \\
\hline Ankle & 06 \\
\hline Metacarpophalangeal & 06 \\
\hline Proximal interphalangeal & 05 \\
\hline Metatarsophalageal & 04 \\
\hline Shoulder & 02 \\
\hline Hip & 01 \\
\hline
\end{tabular}

Investigations - ESR was markedly elevated in all patients, 14 of them having values $>100 \mathrm{~mm} / 1^{\text {st }}$ hour. Leucocytosis, ranging from $11.4 \times 10^{9} / 1$ to $78.2 \times 10^{9} / 1$, was observed in 17 patients. Thirteen patients had anaemia with a mean haemoglobin concentration of $9.1 \mathrm{~g} / \mathrm{dl}$. We could not trace the haemoglobin levels of the remaining 5 patients. Thrombocytosis was seen in 11 patients, 4 had normal platelet counts and we were unable to trace the platelet counts of the other 3 patients. Rheumatoid factor and ANA were done in 16 patients and were uniformly negative.

Complications - One patient had steroid induced glaucoma and another had cataract. Eight patients had developed joint deformities, 7 of whom had active disease for more than 4 years and the other for more than 3 years. Seven patients had developed joint ankylosis, all having had active disease for more than 3 years. Apophyseal joint fusion of the cervical spine was seen in 4 patients all of them having had active disease for more than 4 years. Two patients had atlanto-axial subluxation, both having had the disease for more than 7 years. Two patients had developed avascular necrosis of the hips, one of whom had the disease for more than 4 years and the other for more than 7 years. Growth retardation was seen in 10 patients.

Medications - All 18 patients had received prednisolone. Thirteen patients had been on prednisolone throughout their illness whilst 2 had it for half the disease duration. We could not find the duration of steroid use for the other 3 patients. 17 patients have received MTX. In all patients MTX was started by a rheumatologist. Starting point of MTX varied widely as shown in table 4 . 
Table 4

Starting point of MTX

\begin{tabular}{|l|c|}
\hline Starting point of MTX & No. of children \\
\hline At the beginning & 07 \\
\hline 6 mths -1 yr after onset & 04 \\
\hline$>2$ years later & 03 \\
\hline$>5$ years later & 02 \\
\hline
\end{tabular}

This is because the time period between diagnosis and referral varied widely. 6 patients who had aggressive disease despite steroid and MTX had received levamisole. The most popular NSAIDs among this subset of patients were ibuprofen, naproxen and diclofenac sodium. The adverse effects encountered by this group of patients are shown in table 5 .

Table 5

Adverse effects of drugs

\begin{tabular}{|l|c|}
\hline Adverse effect & No. of children \\
\hline Gastric irritation & 08 \\
\hline Haematemesis/melaena & 01 \\
\hline Osteoporosis (x-ray changes) & 02 \\
\hline
\end{tabular}

Course and outcome - Seven patients out of the 18 had their disease going on for more than 5 years. Eleven patients had a cyclical pattern with remissions and flareups. Of the 11 patients, 9 had 2 cycles and 2 had 3 cycles. Only 3 patients were in complete remission after 1 year of onset. There were another 3 patients whose disease was well controlled with drugs. Twelve patients were having active disease despite medications.

\section{Enthesitis related arthritis}

Thirteen patients were included in this category and they accounted for about $20 \%$ of the study population. All were males. There were 12 Sinhalese and 1 Muslim patient. The onset of disease was between $6-11$ years of age. In 9 patients the onset was over 8 years of age. Only 1 patient gave a family history of ankylosing spondylitis.

Clinical features - Eight patients had fever at the onset. 4 patients had high grade fever and 4 had low grade fever. Eight patients reported having early morning joint stiffness. None had hepatomegaly, splenomegaly or lymphadenopathy. Most commonly involved joints were the knees and the commonest enthesitis was plantar fasciitis as shown in table 6 .

Table 6

Joint involvement \& enthesitis

\begin{tabular}{|l|c|}
\hline Joint/Enthesitis & No. of patients \\
\hline Knees & 08 \\
\hline Ankles & 04 \\
\hline Sacroiliac & 03 \\
\hline Metacarpophalangeal & 02 \\
\hline Plantar fasciitis & 08 \\
\hline Achilles tendonitis & 04 \\
\hline Costochondritis & 01 \\
\hline
\end{tabular}

Investigations - Elevated ESRs were seen in 7 patients while 6 had normal ESRs. Nine patients had normal WBC counts, 8 had normal haemogobin levels and 8 had normal platelet counts. HLA-B 27 was done in 10 patients and was positive in 3 .

Complications - Two patients had developed joint ankylosis and one of them had joint deformities. We did not detect growth retardation, eye complications, cervical spine abnormalities or avascular necrosis of hips in this group of patients.

Medications - Five patients had prednisolone, the duration varying from 2 months to 7 months. Eight patients were given MTX, 2 of them for more than 1 year. Eight patients used sulphasalazine, 7 of them for more than 1 year. All patients were on NSAIDs, indomethacin and diclofenac sodium being the most popular choices.

Outcome - This was assessed in 10 patients. Five patients were in remission while the others continued to have active disease.

\section{Pauciarticular onset JIA}

There were 10 patients in this category, accounting for $16 \%$ of the study population. There was a female predominance with 6 females and 4 males. Eight patients were Sinhalese and the other 2 were Muslims. The majority had disease onset between 5-9 years of age. Two patients gave a family history of rheumatoid arthritis.

Clinical features - Four patients had fever at the onset. Three patients had high grade fever which lasted for 2-3 days and 1 patient had low grade fever. Six patients reported early morning joint stiffness. None had hepatomegaly, splenomegaly or lymphadenopathy. Initial pattern of joint involvement is shown in table 7.

Table 7

Initial joint involvement

\begin{tabular}{|l|c|}
\hline Joint/s & No. of patients \\
\hline Knee & 08 \\
\hline Ankle & 02 \\
\hline Wrist & 02 \\
\hline Proximal interphalangeal & 01 \\
\hline Elbow & 01 \\
\hline Shoulder & 01 \\
\hline Metatarsophalangeal & 01 \\
\hline
\end{tabular}

Investigations - Eight of the 10 patients had high ESRs. Normal WBC counts were seen in 7 patients whilst 3 patients had leucocytosis. No patient had anaemia. Rheumatoid factor was done in 6 patients and was positive in one. ANA had been done in all 10 patients and was negative in 9 and equivocal in one.

Complications - Three patients had developed joint deformities. Growth retardation was seen in 3 patients of whom 2 had had active disease for more than 2 years. One patient had anterior uveitis but was negative for ANA. 
Medications -Eight patients had been given prednisolone. 5 patients were given methotrexate, 4 of whom had it for more than a year. One patient was on sulphasalazine for 8 months.

Outcome - Only 2 patients were in remission after withdrawal of all drugs. After 1 year of onset, 3 patients were asymptomatic but on NSAIDS. Another 2 patients were under control with disease modifying anti rheumatic drugs (DMARDS). Three patients had active disease even after 2 years of onset despite NSAIDS and DMARDS.

\section{Discussion}

\section{Polyarticular onset JIA}

This occurs in $30 \%$ of all patients with JIA, the majority of them being females ${ }^{1}$. These observations were true for our patients. The onset occurred from 1-11 years. However the majority had onset between 4-8 years of age. Four (20\%) patients who gave a family history of rheumatoid arthritis were a distinct group. They were all females and had their disease onset between 7-8 years of age. Fever can be observed in one-third of patients. It is generally low grade, or a high grade fever often of short duration. In very young children, a transient rash lasting 1 or 2 days can occur. Generally, there is no lymphadenopathy, hepatosplenomegaly, or visceral involvement ${ }^{1}$. However, in our study, 75\% had fever and two of them had high fever $\left(>39^{\circ} \mathrm{C}\right)$ for more than 1 month. Knee, wrist, ankle and small joints of hands were the commonest joints involved initially. In polyarticular onset JIA, ESR, WBC and platelet count are non specific i.e. they can be normal or elevated ${ }^{1}$. However, in our study, all patients had high ESR and more than half had ESR of more than $100 \mathrm{~mm} / 1^{\text {st }}$ hour. Further, leucocytosis was seen in more than half of them. Patients who are positive for rheumatoid factor have their disease onset after 8 years and their disease would progress rapidly ${ }^{1,2}$. This observation is true for our patients too. Presence of ANA does not correlate with disease subtype or disease activity or with the presence of chronic anterior uveitis. They do not help in the diagnosis either ${ }^{1,2}$. These two tests were done in more than $80 \%$ of our patients at the onset of the disease although the rationale of performing these two expensive tests on patients with polyarticular onset JIA is questionable. A large majority had developed growth retardation. More than half of patients who had active disease for more than 2 years had joint deformities and ankylosis. Two patients each had eye complications and cervical spine fusion. Avascular necrosis of hip was seen in one patient. The presence of complications in a large number of patients indicate the seriousness of the illness. Therefore polyarticular onset JIA has to be treated aggressively from the beginning. A significant number of patients were given steroids for unacceptably long periods. The main reason was that these patients had been treated for long periods by nonrheumatologists with NSAIDS + steroids combination before they were referred to the rheumatology clinic. Regarding methotrexate, therapy, all but one patient were started on methotrexate by this clinic and this contributed to the relatively shorter duration of methotrexate therapy when compared to that of steroids. Non-rheumatologists seem to be reluctant to start methotrexate which should be the number one drug for moderate to severe cases of polyarticular onset JIA

\section{Systemic onset JIA}

This accounts for $10-20 \%$ of all patients with JIA ${ }^{1}$. In our study group they accounted for about $30 \%$. There is an equal sex incidence with females more commonly affected when disease begins after the age of 10 years ${ }^{1}$. In contrast, there was a male predominance among our patients, M: F ratio being almost $3: 2$. The onset is under 5 years of age in two-thirds of patients and the mean age at onset is 4-6 years ${ }^{1}$. This observation is compatible with our series where 17 of 18 patients had onset under 6 years of age and 3 to 4 years was the commonest age of onset. All our patients had high fever which is the hallmark of this disease. Eight had high fever going on for more than 2 months. Majority had hepatomegaly, splenomegaly and lymphadenopathy. It is significant that we could not find a single patient with serositis either clinically or with imaging studies such as $\mathrm{x}$-ray, $2 \mathrm{D}$ echo or ultra sound scan. The most commonly involved joints (at the initial stage) were knees and ankles. Wrists, cervical spine, metacarpophalangeal and proximal interphalangeal joint involvement were also common. One patient had hip joint involvement and initial hip joint involvement is not uncommon in systemic onset JIA ${ }^{1,2}$. ESR was elevated in all patients with more than two-thirds having ESR of more than $100 \mathrm{~mm} / 1^{\text {st }}$ hour. Leucocytosis \& thrombocytosis are also hallmarks of systemic onset JIA, so much so that normal counts should always raise suspicion about the diagnosis ${ }^{1}$. In our study group, almost all had leucocytosis. Majority had thrombocytosis even though 4 patients had normal platelet counts. ANA and rheumatoid factor are rarely found in systemic onset JIA and their presence should prompt consideration of alternative diagnoses for e.g. Sjogren's, systemic lupus erythromatosus, mixed connective tissue disease and early onset adult type rheumatoid arthritis ${ }^{1,2}$. These two tests were done routinely and at the onset of the disease in 16 of our patients and all had negative results raising doubts about the rationale of performing these two expensive tests on patients with systemic onset JIA. Patients who had active disease for more than 4 years had developed complications, ranging from joint deformities and ankylosis to avascular necrosis of hips and atlanto-axial subluxation. Further, more than half these patients had growth retardation. The high incidence of complications again highlights the seriousness of the disease and the need to treat these children aggressively from the beginning. Twelve $(67 \%)$ patients were having active disease after 1 year of onset and five of them even after 5 years. This again shows the gravity of the illness. Regarding drug treatment, steroids are used in high doses in systemic onset JIA (prednisolone 1$2 \mathrm{mg} / \mathrm{kg} /$ day) ${ }^{1,2}$. More aggressive therapy should be initiated soon after the child is stabilized in order to facilitate early withdrawal of steroids ${ }^{5,6}$. However, 13 $(72 \%)$ of our patients had been on steroids for more than 
half of their disease duration. MTX was started much later (more than 6 months) in half our patients. In all patients MTX was started by the rheumatologist and 7 patients who were referred early in the course of disease had their MTX started at the beginning (thereby reducing the dose and duration of steroids).

\section{Enthesitis related arthritis}

This is a disease of male patients and onset occurs $>8$ years of age ${ }^{1,2,3}$. Our study confirms this observation. Family history of ankylosing spondylitis was given by only 1 patient. Systemic toxicity is absent at the onset and laboratory features will confirm this i.e. ESR, C-reactive protein (CRP) and blood counts would be normal in enthesitis-related arthritis. However, majority of our patients reported fever at the onset and about one-third of them had high fever. Knee was the commonest joint involved followed by ankle and sacro-illiac joint. Though majority had normal blood counts, ESR was raised in half. Another salient feature is that only 3 out of 10 patients had positive HLA-B27. Two of 13 patients had developed ankylosis of affected joints. Growth retardation, eye complications, cervical spine abnormalities and avascular necrosis of hips, which were seen in systemic and polyarticular types, were not detected in this sub-set of patients. When compared to systemic and polyarticular types, steroids were used less frequently and for shorter periods in these patients. However, majority needed disease modifying drugs for control of the disease, the most popular being MTX and sulphasalazine.

\section{Pauciarticular JIA}

This is said to be the commonest type of JIA accounting for $40-50 \%$ of all patients ${ }^{1}$. However, it accounted for only $16 \%$ of our study population. This may be because it is a milder form of arthritis and less cases are referred to the rheumatologist. There are distinctive characteristics which differentiates this from other types of arthritis. They include the predilection for preschool age girls, the involvement of large joints (excluding the hip and shoulder), the frequent occurrence of chronic anterior uveitis and the presence of ANAs ${ }^{1,2}$. The F: $\mathrm{M}$ ratio is $4: 1$ and the age at onset peaks between 1-3 years ${ }^{1}$. However, in our series, the F: M ratio was $3: 2$ and $50 \%$ had their onset between 7-12 years of age. Systemic features are characteristically absent ${ }^{1}$. In our group 3 patients had high grade fever, one had low grade fever and 6 had morning joint stiffness. ESR, CRP and blood counts are normal in these patients ${ }^{1,2}$. However, the majority of our patients had raised ESR (but not to the level seen in systemic onset JIA). Leucocytosis was seen in 3 patients. ANA is said to be present in $40-70 \%$ of patients ${ }^{1,2}$. In our series only one patient was positive for ANA and that too was not a significant titre. Three patients each had growth retardation and joint deformities which is again unusual for this type of disease. Regarding medications, 8 patients had received steroids, 4 for more than 6 months, to control the disease. Five patients were given methotrexate, 4 of them for more than a year. This again points towards a severe form of arthritis. Only 2 patients were in remission. Five patients continued to take medicines to keep their disease under control more than one year after the onset. Another 3 persons were having active disease even after 2 years (of onset) despite treatment.

\section{References}

1. Maddison P J, Isenberg D A, Woo P, Glass D N, editors, Oxford Text Book of Rheumatology $2^{\text {nd }}$ ed. New York: Oxford University Press 1998: 1099 143.

2. Gunatillaka K A N. Rational approach to the child with juvenile idiopathic arthritis. Sri Lanka Journal of Child Health, 2003; 32: 105-8.

3. Foster H E. Chronic arthritis in children and adolescents. Medicine 2002; 30 (8):34-9.

4. Wijayaratne L S- Juvenile idiopathic arthritis - a new classification, Sri Lanka Journal of Child Health 2001; 30:94-5.

5. Wallace C A. The use of methotrexate in childhood rheumatic diseases. Arthritis Rheumatism 1998; 41:381.

6. Emery H M. Treatment of juvenile rheumatoid arthritis - current opinion. Rheumatology 1993; 5:629. 